\title{
FAST INGREDIENT QUANTIFICATION IN MULTIGRAIN FLOUR MIXES USING HYPERSPECTRAL IMAGING

\author{
Carolina Blanch-Perez-del-Notario ${ }^{1,2}$, Wouter Saeys ${ }^{1}$ and Andy Lambrechts ${ }^{2}$
}

\author{
${ }^{1}$ Imec, Kapeldreef 75, 3001, Leuven, Belgium \\ ${ }^{2}$ KU Leuven, Department of Biosystems, MeBioS, Kasteelpark Arenberg 30, 3001, Leuven, \\ Belgium
}

\section{ABSTRACT}

In the past decade, hyperspectral imaging has emerged and matured into one of the most powerful and rapidly growing methods for non-destructive food quality analysis and control. This paper demonstrates fast and automatic quantification of food ingredients with hyperspectral imaging in the visible and near-infrared range in combination with chemometrics and image processing techniques. The application of ingredient quantification in bread flour recipes requires high spatial resolution in addition to spectral discrimination power. Our results show that automatic and accurate quantification of all ingredients can be done, reaching pixel discrimination accuracies above $90 \%$ and ingredient quantification errors within the required $1 \%$ absolute error in weight. The classification accuracy obtained using 15 wavebands on the test images is around 15\% higher than what was obtained with colour imaging.

Index Terms - Hyperspectral imaging, VNIR spectral response, spatial processing, seed abundance estimation.

\section{INTRODUCTION}

Multigrain bread is a type of bread prepared with two or more types of grain. Grains used include barley, flax, millet, oats, wheat, and whole-wheat flour, among others. Some variants also include edible seeds in their preparation, such as flaxseed, quinoa, pumpkin seeds, and sunflower seeds. Sharkma et al (2008) have shown that multi-grain bread has higher nutritional value in proteins, fibers, antioxidants when compared to traditional wheat bread. Other researchers have shown that whole grain bread has been shown to have an impact on reducing cholesterol and triglycerides in the body (Giacco, 2010; Talaei, 2013). This makes it a healthy alternative to traditional wheat bread. Due to the uptake of the healthy lifestlyle concept, multigrain read is becoming increasingly popular with consumers and it is becoming a main driver for growth in the bread segment (Bakery products market- growth, trends and forecast 2019-2024).

Flour milling and bread baking have become sophisticated processes with a high level of process technology, high volume, and efficient production. Quality Control, whether on-line or through sampling and laboratory analysis, is therefore becoming ever more important to achieve consistent ingredients, process conditions and products so as to guarantee its nutritional value and maintain customer satisfaction. To this end, several standard quality control methods are described ranging from wheat kernel inspection to chemical property analysis such as moisture or ash content (D. Shelton et al., 2004).

In the case of multigrain flour mixes, it is important to verify that the different ingredients are present in the desired range. This requires the analysis of a subsample of the flour batch where all seed and non-seed ingredients have to be discriminated and then quantified to assess whether their presence in the mix is according to the recipe. This quality inspection is currently done manually, and the procedure involves manual sieving with multiple sieve sizes to separate the different seeds/ingredients and then weight each sorted ingredient independently. This is very time-consuming and as a result only a very small amount of the flour batch is typically inspected.

The commercially available automatic seed counting machines cannot solve this quantification problem, because these devices only measure one seed type at a time. Therefore, to quantify the ingredients in a multi-grain mix with these systems the seeds should be sorted first and measured separately. This is a cumbersome process. Moreover, more heterogeneous and fractionable ingredients such as wheat or barley flakes may not be addressed with these machines.

Machine vision is already widely used for food quality analysis (K. Patel et al., 2012). More specifically, in the flour industry, machine vision with color imaging has been used for cake crumb color analysis according to the type of flour used (F. Rezaghli et al., 2017) or for wheat kernel inspection, and brown versus yellow flax seed sorting (T.C. Pearson et al., 2013). (F. Kurtulmus et al., 2015) reported an accuracy of $84 \%$ in the identification of different pepper seed varieties for machine vision with color cameras. Similarly, P.M Szczypiński et al, (2015) classified barley seed varieties based on shape, color and texture features with accuracies between 67 and 86\%. 
Hyperspectral imaging, combining spectroscopy and imaging, adds an extra dimension to machine vision techniques by providing images at a larger number of more narrow wavebands. Thanks to its higher discriminating power, it is gaining attention as a non-destructive, real-time detection tool for food quality analysis and control (Amigo et al., 2013; H.Huang et al., 2014; D. Lorente et al., 2011). Specifically for flour inspection, it has been shown to provide peanut contaminant detection in flour (A.Herrero et al., 2015; X. Zhao et al., 2018) and measurement of chemical properties such as protein (Caporaso et al., 2018), sugar or azodicarbonamide (Wang et al, 2018). Most reported studies on flour inspection with hyperspectral technology focused on the SWIR range (1000 to $2500 \mathrm{~nm}$ ), which contains the most detailed chemical information.

Additionally, the use of hyperspectral imaging in the VNIR range has been shown to increase further the discrimination power for seed quality assessment. Ma et al. (2015) obtained a classification accuracy of $97 \%$ for sunflower seed quality inspection with hyperspectral imaging in the VNIR range. Vu et al. (2016) reported that adding VNIR spectral information to shape-based features increased the classification accuracy for rice seed varieties up to $84 \%$. Boelt et al. (2018) reported on the application of hyperspectral imaging for sugar beet seed maturity level discrimination with 95\% accuracy. Finally, Carstensen (2018) proposed a spectral imaging system in the VNIR range for discrimination of durum wheat versus common wheat seed reaching $99 \%$ accuracy. Most previous studies focused on viability or quality prediction for one type of seed and show the added value of hyperspectral imaging (combining spectral information and spatial/shape information).

Feng et al. (2019) presented an extensive review on existing studies with hyperspectral imaging for seed quality and safety inspection. Most of the reviewed work on seed discrimination with hyperspectral imaging uses the spectra either from ROIs in bulk samples (Zhang et al., 2012, Kong et al., 2013) scanning one seed type per image, or by extracting the mean spectrum from a ROI of each individual seed (Sun et al., 2016). Choudhary et al. (2009) and Manley et al. (2011) worked with spectra at pixel level, but with lower resolution imaging in the SWIR range. In all reviewed studies, each image was of a specific seed class/variety and different types were never mixed in one single image. Therefore, the aim of this study was to identify multiple seed and non-seed ingredients in one scan, while the spectra of each individual pixel are used for discrimination.

To be able to accurately discriminate these similar looking seed or grain ingredients it is expected that more spectral information is required than the R, G and B channels used in color imaging. Moreover, we focus here on an industrial inspection application that requires high spatial resolution since we need to inspect sufficient amount of multi-ingredient flour mix (imaging enough seeds/flakes per scan) and this mix often consists of small seeds (e.g poppy, chia...). For this reason, it was assumed that the additional chemical information provided by the SWIR range would not be as relevant as having the high spatial resolution available in the VNIR range.

In addition, for industrial inspection we need high acquisition speed to sample enough product, while retaining enough discrimination power. From previous studies, only Carstensen et al., (2018) addressed fast and highresolution imaging in the VNIR range. However, this was done for a maximum of 20 spectral bands and to discriminate between only two wheat varieties.

In contrast, in our industrial application we need fast acquisition of multiple seed and non-seed ingredients simultaneously. These seeds will be of different varieties and adjacent to each other, which further challenges accurate discrimination and quantification requiring high spatial and spectral resolution. Moreover, to be able to estimate component abundance in weight we need to assume similar thickness for some ingredients such as flakes. However, in practice, we can expect a variability of around 30\% in the flake thickness, as reported for oat (Rosentrater et al., 2018) or for other types of flakes (Mathison et al., 1997), (Schwandt et al., 2017).

To the best of our knowledge our study is the first to focus on the use of hyperspectral imaging for accurate seed type discrimination and quantification in a multiple seed and flake type mix in industrial inspection.

\section{MATERIALS AND METHODS}

\subsection{Hyperspectral imaging setup}

Figure 1 shows the imaging system used: a snapscan camera (Pichette et al., 2017), which offers both high spatial resolution (up to 7Mpixels) and high spectral resolution (150 bands) over the wavelength range of 470 to $900 \mathrm{~nm}$. Thanks to its internal translation stage, there is no need for an external scanning movement. Scanning is handled internally, performing data-set acquisition as easily as with a snapshot camera. Full hyperspectral images can be acquired in less than 10 seconds. 


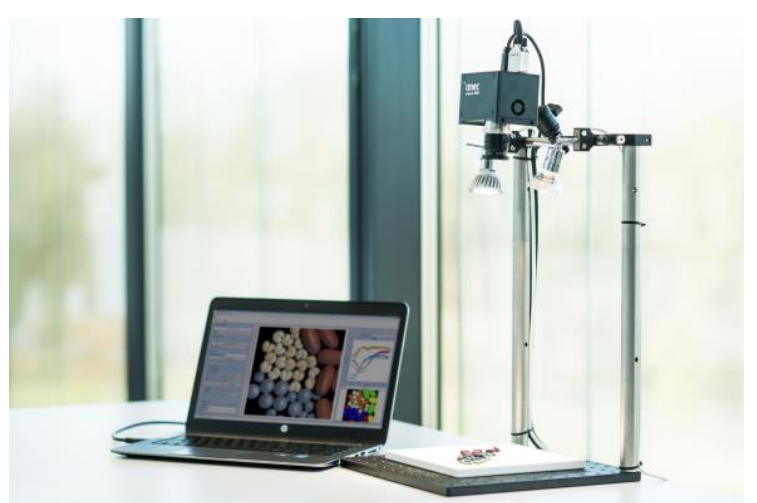

Figure 1: Imec Snapscan System with Snapscan camera USB-connected to laptop

\subsection{Multigrain flour samples}

Table 1 shows two typical flour formulas that we replicate in our tests. We can see that the most abundant ingredient is wheat flour, while the seed ingredients are present in small percentages. In this respect, the quality inspection of ingredients requires that the estimation error on the mass fraction is below $1 \%$.

Note that the relative abundance of wheat flour cannot be estimated based on a hyperspectral image since no 3D or depth information from the layer of wheat flour can be obtained. Therefore, it was decided to sieve the flour away from the flour-mix. This allows to weight the flour separately and prevents flour from covering other ingredients and making them undetectable for the hyperspectral camera.

Table 1a: Flour mix formula 1

\begin{tabular}{|l|l|l|l|l|l|l|l|l|l|}
\hline Ingredients & $\begin{array}{l}\text { Sunflower } \\
\text { seeds }\end{array}$ & $\begin{array}{l}\text { Wheat } \\
\text { bran }\end{array}$ & $\begin{array}{l}\text { Yellow } \\
\text { millet }\end{array}$ & $\begin{array}{l}\text { Red } \\
\text { millet }\end{array}$ & $\begin{array}{l}\text { Poppy } \\
\text { seeds }\end{array}$ & $\begin{array}{l}\text { Sesame } \\
\text { seeds }\end{array}$ & $\begin{array}{l}\text { Brown } \\
\text { linseed }\end{array}$ & $\begin{array}{l}\text { Corn } \\
\text { crispy }\end{array}$ & $\begin{array}{l}\text { Wheat } \\
\text { flour }\end{array}$ \\
\hline Percentage (\%) & 1.75 & 4.00 & 2.00 & 2.00 & 1.75 & 2.00 & 2.00 & 1.75 & 84.75 \\
\hline
\end{tabular}

Table 1b: Flour mix formula 2

\begin{tabular}{|l|c|c|c|c|c|c|}
\hline Ingredients & Oat flakes & Cracked wheat & Barley flake & Linseed & Corn crispy & Wheatflour \\
\hline Percentage (\%) & 5.00 & 2.50 & 5.00 & 2.50 & 3.75 & 81.25 \\
\hline
\end{tabular}

We created five flour mix samples of $5 \mathrm{~g}$ for formula 1 by weighting the different ingredients to respect their mass fractions listed in Table 1a and $\mathrm{b}$. The error on the weight measurement from our device being around $0.01 \mathrm{gr}$. We then mixed the ingredients in a petri dish, sieved away the flour and imaged the petri dishes with the hyperspectral camera. For formula 2 we also created five mixes, but in this case, we varied this time the absolute weight of non-seed ingredients. An area of roughly 2000x2000 pixels (4Mpixels) was imaged to match the size of the petri dish.

\subsection{Preprocessing and classification methods}

We first define a ground truth by creating masks selecting different seed types. From the masks 1000 spectral pixels are randomly selected, corresponding to roughly $20 \%$ of the available ingredient pixels in the full image. Out of these 1000 spectra per ingredient type, 50\% were randomly selected for training and the remaining 50\% for validation. These sets were then used to compare the different feature selection and well-known classification methods in the PerClass software tool (PerClass BV 2008-2019). The classification performance is then validated on the full image and on four independent images.

As feature selection method we used either none, Linear Discriminant Analysis (LDA) or Principal Component Analysis (PCA) (Smith et al., 2002). For PCA we selected the number of components that captured $99 \%$ of the variance, this allowed us to keep 9 PCA components and provided better results than targeting lower variance or higher (100\% retains all bands).

The following classification methods available in the PerClass software tool were tested for discriminating the different ingredients based on their spectra:

- Linear Discriminant Analysis (LDA) or Linear Discriminant Classifier (LDC) (Naes et al., 2004), assuming normal densities for our classes. 
- Quadratic Discriminant Classifier (QDC), also known as Quadratic Discriminant Analysis (Naes et al., 2004) assuming as well normal densities for our spectral classes.

- $\quad$ Support Vector Machines (SVM) (Hsu et al., 2016): with an RBF Kernel selection and a grid search of sigma and $\mathrm{C}$ parameters.

- $\quad$ Random forest (RF) (Yiu, 2019) with the default value of 20 trees, considering a subset of $20 \%$ of features at each node

- $\quad$ Artificial Neural Networks (ANN) (Han et al., 2012): a feed-forward network with 30 hidden neurons was selected and 5000 iterations allowed.

\subsection{Image spatial post-processing}

Some of the recipe ingredients such as all seed types (linseed, sunflower, poppy, millet...) are very homogeneous in shape and size. Therefore, both an average number of pixels per seed and an average weight per seed can be estimated for a given camera-object distance, lens type and image spatial resolution. These are summarized in our case in Table 2.

Table 2: Pixel-weight relation for different seeds

\begin{tabular}{|l|l|c|}
\hline Ingredient & Weight/seed (g) & Pixels/seed \\
\hline Sunflower seeds & $0.03-0.05$ & 3000 \\
\hline Yellow millet & 0.0066 & 330 \\
\hline Red millet & 0.0066 & 400 \\
\hline Brown linseed & 0.0066 & 1000 \\
\hline Sesame seeds & 0.0014 & 400 \\
\hline Poppy seeds & 0.0006 & 120 \\
\hline Corn crispies & 0.0175 & 2000 \\
\hline
\end{tabular}

171

172

173

174

175

176

177

178

179

180

181

182

183

184

185

186

187

188

189

190

191

192

193

194

195

196

197

198

199

200

201

202

203
We used formula 1 (Table 1a) to study seed discrimination and quantification, while formula 2 (Table 1b) was used to study the quantification of non-seed ingredients. For each formula we used one image for training and four images as independent test set for validation. Non-seed ingredients, such as wheat bran or even barley flakes, are heterogeneous in size and shape. This makes it difficult to obtain an average weight or pixel number. Their abundance estimation will be explained in more detail in the following section.

\subsubsection{Estimation of abundance per seed ingredient}

Since the seeds are very homogeneous in size and shape, we assume that its abundance in grams can be safely estimated from the number of seeds present in the classified/labelled image. As we will later see, the presence of miss-classified pixels can make the seed count challenging. In order to correct these miss-classifications and we perform some additional spatial imaging post-processing step. This step cleans the classified mask images and helps obtain a more accurate seed count.

The additional image processing proposed is done in Matlab 2015 (The Mathworks, Natick, MA, USA) and consists of a combination of the following common morphological operations in this order:

- Area open: the operation of area opening removes from a binary image all connected components (objects) that have fewer than ' $\mathrm{P}$ ' specified pixels, defining the cluster size to be removed. The default connectivity for pixels to belong to an object is 8 for two dimensions.

- Area fill: a morphological operation with the purpose to fill small pixel holes in objects/seeds by setting a pixel to 1 if five or more pixels in its $3 \times 3$ neighborhood are 1's.

- Object count: this operation counts all 8-connected objects (Ghuneim, Pixel connectivity) in the image. With this final step the total seed count is extracted.

The first step in our spatial post-processing consists of removing clusters of miss-classified pixels. Our ' $\mathrm{P}$ ' parameter tuning allows to specify the object size to be considered as miss-classification and removed from the image. Parameter ' $\mathrm{P}$ ' is related both to the expected size of the seed as well as to the proportion of expected missclassified pixels. On one hand, this parameter must be roughly below 50\% the pixel size of the target seed to avoid eliminating partially eroded seeds or half seeds present in the image. This is particularly the case for sunflower and linseeds, which tend to fracture. On the other hand, the ' $\mathrm{P}$ ' parameter must be large enough to safely eliminate miss-classified shades/borders coming from non-target seeds. This is typically obtained with values around 10- 
$20 \%$ the pixel size of the target seed. In the case of sesame and millet seeds, where there are more miss-classified pixels but no fractured seeds, values up to $40 \%$ the size of the seed were found to be suitable.

Table 3 shows the selected ' $\mathrm{P}$ ' parameter, found experimentally and validated over several independent images.

Table 3: ' $P$ ' parameter selection versus target seed size

\begin{tabular}{|l|l|c|c|c|c|l|l|}
\hline & Sunflower & Corn & Linseed & Sesame & Millet & Red millet & Poppy \\
\hline Average size in pixels & 3000 & 2000 & 1000 & 400 & 330 & 400 & 120 \\
\hline 'P' parameter & 600 & 600 & 300 & 150 & 150 & 150 & 50 \\
\hline
\end{tabular}

The estimation of some seed types requires additional post-processing. This is the case of millet, where a higher number of miss-classifications occur, and an additional step is added to count only circular shaped objects.

- Find circular shapes: This algorithm, based on the circular Hough transform, detects circular shapes within a predefined diameter range. For our images, the range was set from 8 to 20 pixels.

Another seed that requires an additional post-processing is the poppy seed. Its spectral discrimination is very accurate, but their seeds often cluster together and can be mistakenly counted as one single object or seed. Therefore, instead of an object count we use in this case:

- Pixel-count based estimation: this step divides the total number of pixels labelled as 'poppy seed' by the average size of a poppy seed (here 120 pixels). This quotient provides then a more accurate estimation of the total number of seeds when clustered.

\subsubsection{Estimation of non-seed ingredients}

The size and shape heterogeneity of non-seed ingredients such as flakes or bran (from oat, barley or wheat) makes it more challenging to estimate the weight/percentile abundance based on the number of flakes or pieces. In this case, considering the number of total pixels labeled as a specific cereal gives a better indication of its actual weight. To do so we assume a similar thickness for these ingredient flakes. Since multiple flakes are imaged simultaneously, we will see that on average a good estimation of the weight in grams can be obtained from the pixel count.

A total of five petri dishes with different abundances of non-heterogeneous ingredients (such as flakes), corresponding to the flour mix in formula 2, were also imaged and then classified. One image was used for training and the remaining four for testing.

\subsection{Waveband selection}

To identify the most discriminative bands in the VNIR wavelength range a Genetic Algorithm was used (Holland, 1992), in combination with our best performing classifier: LDA as feature selection followed by QDC. The aim was to find a near-optimal number of reduced bands providing still high classification accuracy. This information could be used for instance to enable customizing the hyperspectral sensor or alternatively to process less bands and simplify the classification process.

The behavior of the Genetic Algorithm is schematically illustrated here and in Figure 2 (Blanch et al., 2019):

- For a desired number of subset bands/wavelengths (e.g 3 ...) an initial 'population' of individuals is created. Every 'individual' consists of a specific set of random band selections (e.g. [630nm, 770nm, 900nm]. An individual with a uniform selection is also included in the initial population.

- For each individual solution (band selection) the fitness function of the Genetic Algorithm is computed as the mean classification accuracy obtained for that band subset on a fixed training and testing set.

- The Genetic Algorithm iterates for a given number of iterations. In each generation (iteration) the best performing 'individual' or band subsets are kept breeding new generations.

Our LDA+QDC classifier is trained on 50\% of our random sample selection and tested on the remaining 50\%. 


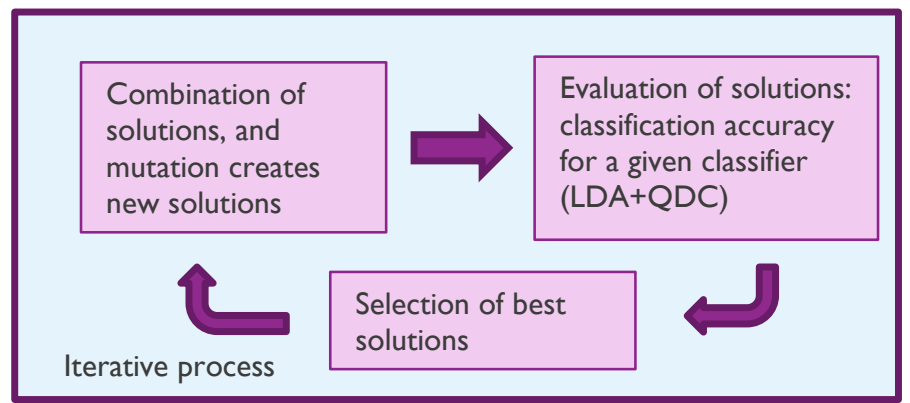

Figure 2: Schematics of Genetic Algorithm

\subsection{Comparison with RGB image discrimination accuracy}

To benchmark hyperspectral imaging in the VNIR range $(460-900 \mathrm{~nm})$ with respect to regular color imaging cameras we create RGB images from our hyperspectral image. This way, we can compare both imaging systems under the same system conditions: illumination, lens, and identical sample selection.

To obtain the corresponding RGB image from the hyperspectral image we use XYZ parameter computation (Goodman, 2012) from our hyperspectral image. The CIE XYZ color space is a device-invariant representation of color. From the given spectrum, $S$, the illuminant function $I$, (here assumed $\mathrm{E}$ standard of theoretical equal energy radiator (Goodman, 2012)) and the CIE color matching functions, we can obtain X, Y and Z color values. We can then transform these X, Y, Z values to RGB color components (International Colour Consortium 1999, sRGB).

\section{RESULTS AND DISCUSSIONS}

Figure 3 shows the mean reflectance spectra of the different ingredients in Table 1, where we can see that some ingredients have a visually similar mean spectrum (e.g wheat bran and sesame). Table 4 summarizes the classification accuracies obtained for the validation set of said ingredients. The different feature selection and classification methods are compared using the exact same set of training and validation samples.

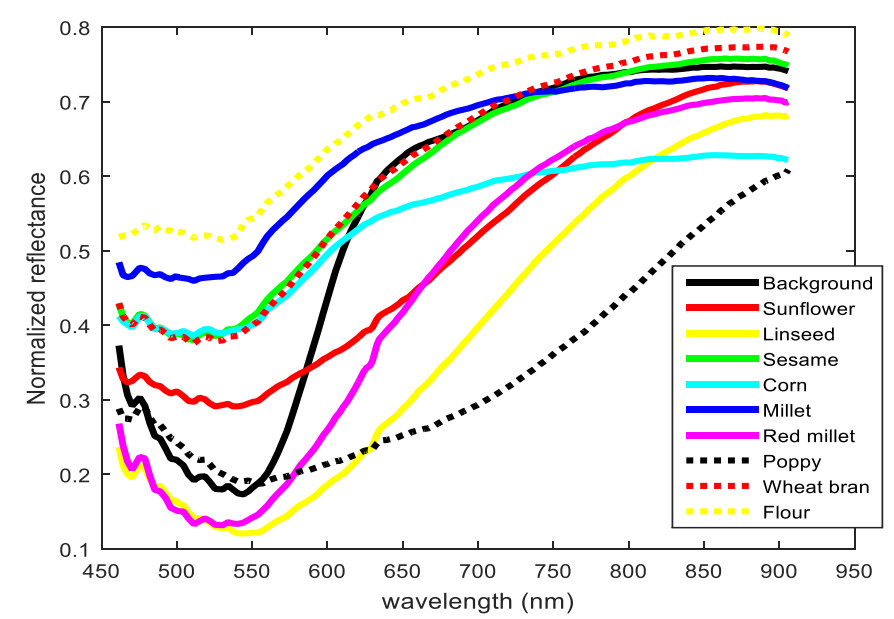

Figure 3: Mean reflectance spectra of ingredients

Table 4: Comparison of feature selection and classifiers performance at pixel level

\begin{tabular}{|l|l|l|l|}
\hline Feature selection & Classifier & $\begin{array}{l}\text { Minimum } \\
\text { accuracy (\%) }\end{array}$ & $\begin{array}{l}\text { Mean } \\
\text { accuracy (\%) }\end{array}$ \\
\hline PCA & QDC & $66.8 \%$ & $91.8 \%$ \\
\hline PCA & SVM & $68.1 \%$ & $91.4 \%$ \\
\hline LDA & LDC & $61.4 \%$ & $90.3 \%$ \\
\hline LDA & QDC & $\mathbf{7 5 . 0 \%}$ & $\mathbf{9 3 . 0 \%}$ \\
\hline LDA & SVM & $49.9 \%$ & $87.6 \%$ \\
\hline LDA & RF & $68.6 \%$ & $92.0 \%$ \\
\hline None & ANN & $53.4 \%$ & $89.1 \%$ \\
\hline
\end{tabular}


We can see that the combination of Linear Discriminant Analysis (LDA) as feature selection step and Quadratic Discriminant Analysis (QDC perClass) as classification method outperforms all other methods in terms of minimum and mean accuracies. It is interesting to notice for instance that PCA as feature selection method provides in this case a significant lower accuracy than LDA as feature selection to QDC. However, for the SVM classifier it is PCA that performs best. While the mean accuracy is comparable for all classification methods, it is for the challenging classes (minimum class accuracy) where we can see LDA+QDC clearly outperforming all other methods. Moreover, its computational time is lower than for more advanced classifiers such as Support Vector Machines (SVM) or random forest (RF). The normalized confusion matrix for LDA+QDC classifier and the seed ingredients given in Table 1 is shown in Table 5 below.

\section{Table 5: Confusion matrix for LDA+QDC}

\begin{tabular}{|c|c|c|c|c|c|c|c|c|c|c|c|c|c|}
\hline \multirow{3}{*}{ Background } & & \multicolumn{12}{|c|}{ Decisions } \\
\hline & & $1: 1$ & $2: 2$ & $3: 3$ & $4: 4$ & $5: 5$ & $6: 6$ & $7: 7$ & $8: 8$ & $9: 9$ & $10: 10$ & 11:11 & 1sum \\
\hline & $1: 1$ & 0.99 & 0 & 0 & 0 & 0 & 0.00 & 0 & 0 & 0.00 & 0 & 0.00 & 1.00 \\
\hline Sunflower & $2: 2$ & 0 & 0.98 & 0.01 & 0.00 & 0 & 0.00 & 0 & 0 & 0.01 & 0 & 0 & 1.00 \\
\hline Linseed & $3: 3$ & 0 & 0.02 & 0.97 & 0 & 0 & 0 & 0 & 0 & 0.01 & 0 & 0.00 & 1.00 \\
\hline Sesame & $4: 4$ & 0 & 0.00 & 0 & 0.90 & 0 & 0.01 & 0 & 0 & 0.09 & 0 & 0 & 1.00 \\
\hline Corn & $5: 5$ & 0 & 0 & 0 & 0 & 0.95 & 0.04 & 0 & 0 & 0.00 & 0 & 0 & 1.00 \\
\hline Millet & $6: 6$ & 0 & 0 & 0 & 0.04 & 0.13 & & 0 & 0 & 0.08 & 0.01 & 0 & 1.00 \\
\hline Red millet & $7: 7$ & 0 & 0 & 0.00 & 0 & 0 & 0 & 0.98 & 0 & 0.01 & 0 & 0 & 1.00 \\
\hline Poppy & $8: 8$ & 0 & 0 & 0 & 0 & 0 & 0 & 0 & 1.00 & 0 & 0 & 0 & 1.00 \\
\hline Wheat_bran & $9: 9$ & 0.00 & 0.00 & 0 & 0.09 & 0 & 0.03 & 0 & 0 & 0.86 & 0.03 & 0 & 1.00 \\
\hline Flour & $10: 10$ & 0 & 0 & 0 & 0 & 0 & 0.09 & 0 & 0 & 0.17 & & 0 & 1.00 \\
\hline
\end{tabular}

Values shown are fractions of one, where one is equivalent to $100 \%$ of the pixels correctly classified. The left column shows real class labels while the upper row shows decisions or labels attached by the classifier. Values in the main diagonal mean correctly labeled samples while values outside the diagonal are miss-classifications between classes. We can see that, although classifications accuracies are generally high (over $75 \%$ ) pixel missclassifications can be expected. This way, corn and millet are sometimes miss-classified due to their spectral similarity. This can also happen between wheat bran and sesame. In Figure 4, a small crop of the original image acquired for the ingredients in Formula 1 and the corresponding classified image is illustrated. We can see that, after sieving, some remaining small amount of flour may be detected and classified. We can as well observe how small miss-classifications happen on seed parts, especially for millet and corn, which suffer miss-classifications with each other. These miss-classifications interfere with the individual seed recovery/count required for ingredient quantification and need to be corrected by post-processing.
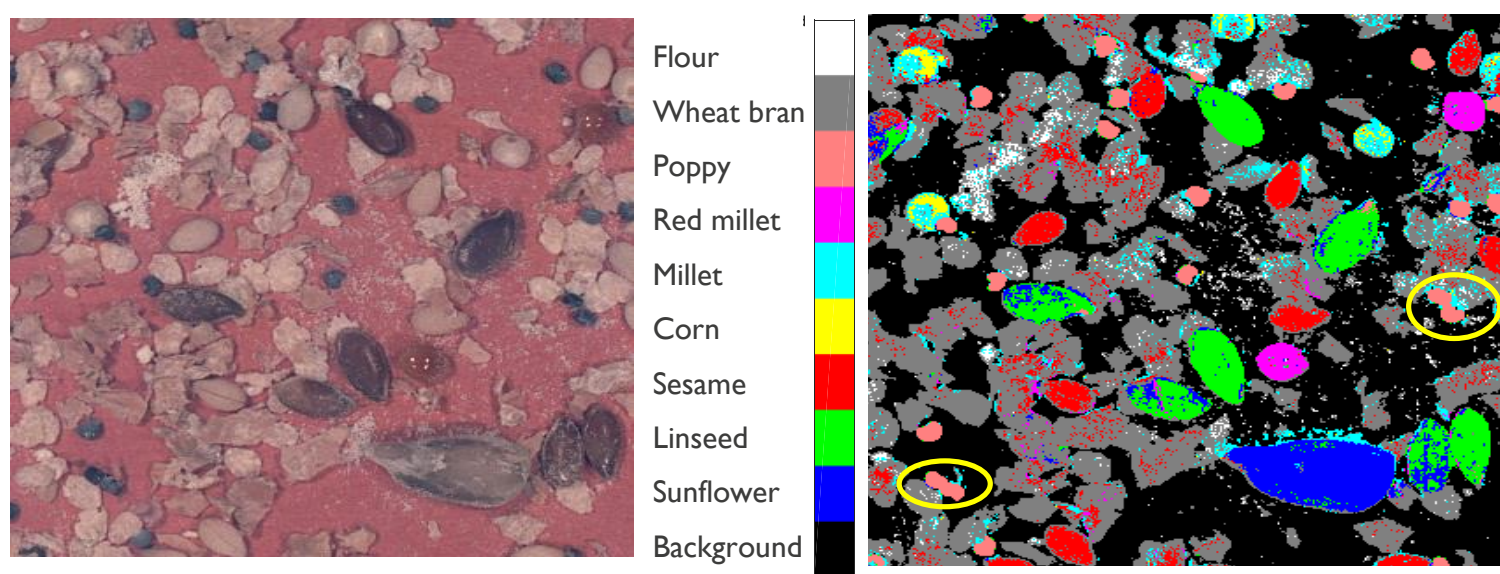

Figure 5 shows the resulting original and spatially post-processed masks for yellow millet seeds. The middle figure shows the mask of pixels classified as millet, where quite some pixel miss-classifications occur. Small miss-classifications are in general efficiently removed by the morphological operations described in Section 2. 
We can see now in Figure 5 (right) that pixels corresponding to the real seeds (four millet seeds) can be identified better. Occasionally, however, some seed portions may be eroded away, or a small cluster of miss-classified pixels may persist. In the case of poppy seeds, the classification accuracy is already high, and fewer miss-classifications are present. However, to deal with seed clusters (see yellow circles in Figure 4), the total pixel count step is required.
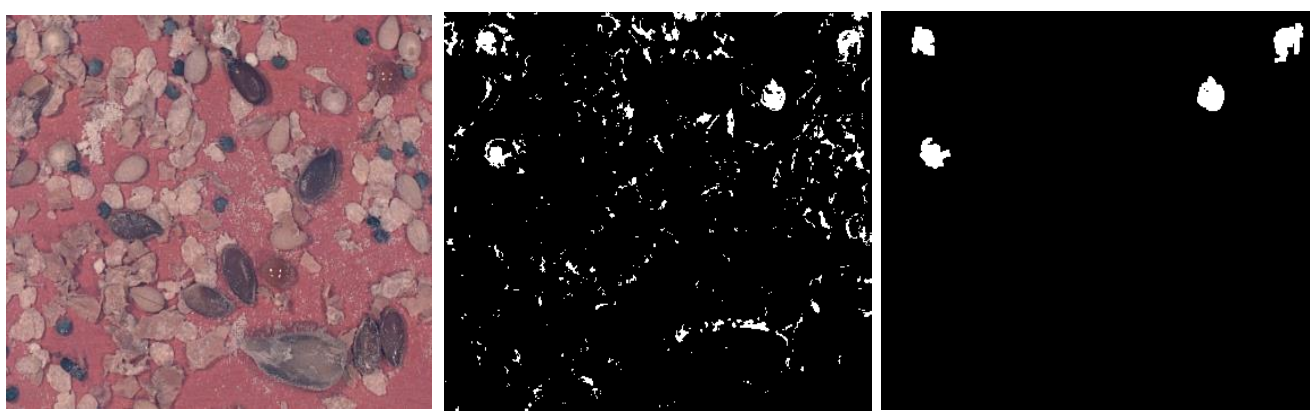

311

\section{Figure 5: Color image of crop (left) and spatial processing of yellow millet (middle and right)}

Table 6 shows the final seed count obtained for the full-size petri dish of Figure 4, as well as the abundance estimation errors in relative and absolute terms. The relative error per ingredient has been computed from the number of estimated and actual seeds. For instance, 15 seeds are detected out of the 16 linseeds, resulting in a $6.25 \%$ relative error. To compute the absolute error, we considered the specific abundance of linseeds in the flour mix. Since linseed constitutes $2 \%$ of the mix, the absolute error has been computed as $6.25 \% * 2 \%$, which corresponds to an absolute error of $0.12 \%$.

We can see in Table 6 that yellow millet has the highest estimation error (16\% relative error). All other seed types are estimated accurately with relative estimation errors below $10 \%$. Our industrial application requires that the absolute estimation error is below $1 \%$. This way, a seed of $5 \%$ abundance in the flour mix can be estimated in the 4-6\% range with up to $20 \%$ relative estimation error. Therefore, for the ingredients in Table 1 , all present at a maximum of $5 \%$, we are within the required $1 \%$ absolute error even in the case of a $20 \%$ relative error.

Table 6: Final seed accuracy for seed ingredients in Formula 1

\begin{tabular}{|l|c|c|c|c|c|}
\hline Ingredient & $\begin{array}{l}\text { Perc } \\
(\%)\end{array}$ & $\begin{array}{l}\text { Actual \# } \\
\text { seeds }\end{array}$ & $\begin{array}{l}\text { Estimated \# } \\
\text { seeds }\end{array}$ & $\begin{array}{l}\text { Relative } \\
\text { error (\%) }\end{array}$ & $\begin{array}{l}\text { Absolute } \\
\text { error (\%) }\end{array}$ \\
\hline Sunflower & 1.75 & 2 & 2 & 0.00 & 0.00 \\
\hline Linseed & 2.00 & 16 & 15 & 6.25 & 0.12 \\
\hline Corn & 1.75 & 5 & 5 & 0.00 & 0.00 \\
\hline Red millet & 2.00 & 14 & 14 & 0.00 & 0.00 \\
\hline Yellow millet & 2.00 & 22 & 26 & 18.18 & 0.36 \\
\hline Sesame & 2.00 & 37 & 34 & 8.10 & 0.16 \\
\hline Poppy seed & 1.75 & 113 & 106 & 6.19 & 0.11 \\
\hline
\end{tabular}

Our classification and quantification method, trained on one image, has been validated over four independent test images from petri dishes of the same formula 1. The results are summarized in Table 7.

Table 7: Final seed accuracy for seed ingredients in Formula 1, average over validation dishes

\begin{tabular}{|l|c|c|c|c|c|c|c|}
\hline \multirow{2}{*}{ Ingredient } & \multirow{2}{*}{$\begin{array}{l}\text { Perc } \\
(\boldsymbol{\%})\end{array}$} & \multicolumn{2}{|c|}{ Relative \% error per validation dish } & Mean relative & $\begin{array}{l}\text { Mean absolute } \\
\text { error (\%) }\end{array}$ \\
\cline { 3 - 7 } & & Dish 1 & Dish 2 & Dish 3 & Dish 4 & error (\%) & 0.00 \\
\hline Sunflower & 1.75 & 0.00 & 0.00 & 0.00 & 0.00 & 0.00 & 0.00 \\
\hline Corn & 1.75 & 0.00 & 0.00 & 0.00 & 0.00 & 0.00 & 0.09 \\
\hline Linseed & 2.00 & 0.00 & 6.25 & 6.25 & 6.25 & 4.68 & 0.07 \\
\hline Sesame & 2.00 & 5.41 & 0.00 & 0.00 & 8.11 & 3.38 & 0.20 \\
\hline Yellow millet & 2.00 & 4.54 & $\mathbf{2 2 . 7 2}$ & 4.54 & 9.09 & 10.22 & 0.16 \\
\hline Red millet & 2.00 & 0.00 & 8.33 & 15.38 & 8.33 & 8.01 & 0.13 \\
\hline Poppy seed & 1.75 & 8.84 & 10.61 & 6.19 & 7.96 & 8.40 & \\
\hline
\end{tabular}

As we can see, all ingredient estimation is below the $1 \%$ absolute error required, except for the more challenging ingredient, yellow millet, in one of the test images (dish 2). In this case, a relative error of $22 \%$ (higher than 20\%) translates in an absolute error above $1 \%$ for seeds with higher abundance than $10 \%$. However, typically seed 
abundance in a flour mix is lower than $10 \%$, in particular for small size seeds such as millet. It is also interesting to note that the relative errors for different images of the same recipe can vary. This is probably related to seed distribution, where some ingredients can end up clustered, occluded or imaged at a different angle. Therefore, by averaging over different images of the same recipe we can also expect to reduce our estimation error, as it can be seen in the mean error over the 4 test dishes in Table 7.

By performing hyperspectral imaging followed by spatial post-processing, and averaging over images, we have achieved relative errors below $10 \%$, equivalent to a discrimination/quantification accuracy over $90 \%$. This high seed discrimination accuracy has been reported as well in literature by combining hyperspectral and texture/spatial information. This way, Sun et al, (2016) also found that combining hyperspectral imaging in the VNIR range and spatial information (such as texture/morphology) achieves the highest discrimination accuracy (98\% versus $86 \%$ for only texture based), for black bean varieties. Similar conclusions are drawn for maize variety discrimination in (Zhang et al., 2012) where combining textural information with VNIR hyperspectral achieves 98.8\% accuracy on 100x100 pixel ROIs from bulk samples. Choudhary et al, (2009) showed 99\% classification accuracy of 8 wheat varieties by combining wavelet texture features from ROIs with hyperspectral data in the SWIR range (900-1700nm). Manley et al, (2011) assessed the impact of kernel topography on pixel spectra from wheat, barley and sorghum kernels, but no classification results are provided.

In our work we have used a high-resolution imager in the VNIR (4Mpx with respect to 0.3Mpx in previous works) to better image multiple seed types in one scan, instead of one single type (or even one single seed) per image. Sun et al. (2016), Zhang et al. (2012) limited their spectral information to the mean spectra from manually predefined ROIs. In contrast to this, we have used pixel-based spectral analysis.

Moreover, we have combined hyperspectral and spatial/shape information in a different way than previous approaches. In previous works this was done by extracting texture features from defined ROIs covering bulk samples (Zhang et al., 2012) or single seeds (Sun et al., 2016), or by more complex wavelet analysis (Choudhary et al., 2009). Instead, we have done this by applying a few morphological steps such as erosion on the already pixel-based classified image. We have shown that this combination of pixel-based spectral analysis and spatial post-processing can reach high discrimination and quantification accuracy.

\section{Non-seed ingredient estimation based on pixel count}

We related the number of pixels labelled by the classifier as a specific ingredient with its absolute weight in grams. Table 8 shows an example of such measured relation for barley flakes and wheat bran in different weights, with a confidence level given for our weight measurement error.

Table 8: Corresponding weight and number of pixels

\begin{tabular}{|l|c|l|c|l|}
\hline Formula 2 & \# pixels (barley flakes) & Weight & \# pixels (wheat bran) & Weight \\
\hline Image 1 & 91277 & $0.54+/-0.01 \mathrm{gr}$ & 207692 & $0.11+/-0.01 \mathrm{gr}$ \\
\hline Image 2 & 51685 & $0.31+/-0.01 \mathrm{gr}$ & 378763 & $0.23+/-0.01 \mathrm{gr}$ \\
\hline Image 3 & 131929 & $0.72+/-0.01 \mathrm{gr}$ & 114861 & $0.09+/-0.01 \mathrm{gr}$ \\
\hline Image 4 & 169292 & $0.91+/-0.01 \mathrm{gr}$ & - & - \\
\hline Image 5 & - & - & 286406 & $0.15+/-0.01 \mathrm{gr}$ \\
\hline
\end{tabular}

In percentual value this error can range from 1 to $10 \%$ error depending on the total weight measured. For all weight and pixel values in Table 8 we obtain an average weight versus average number of pixels ratio. Based on this ratio, we then compute the estimated ingredient weight based on the number of pixels identified in each image. To extract the estimation error, we compare estimated weight and real known weight and compute both the coefficient of determination $R^{2}$ and the mean error incurred with respect to the measured weight, extracted as the $R M S E$ of the estimation errors in percentual value.

Note that the error in the weight estimation can happen due to several factors such as:

- $\quad$ the error on the measurement device

- the fact that ingredients may lay on top of each other (this is particularly the case for wheat bran, which is very light and tends to overlap)

- $\quad$ small variations in flake thickness that may not be averaged out over this quantity of flakes

Table 9 summarizes the weight estimation error incurred for each of the tested non-seed ingredients: 
Table 9: Weight estimation error for non-seed ingredients

\begin{tabular}{|l|l|l|l|l|}
\hline Ingredient & Barley flake & Wheat bran & Oat flake & Cracked wheat \\
\hline Mean weight error estimation & $4.6 \%$ & $13.0 \%$ & $5.6 \%$ & $1.9 \%$ \\
\hline$R^{2}$ coefficient & 0.98 & 0.91 & 0.76 & 0.99 \\
\hline
\end{tabular}

386

387

388

389

390

391

392

393

394

395

396

397

398

399

400

401

402

403

404

405

406

407

408

409

410

411

412

413

414

415

416

417

418

419

420

421

422

423

424

425

426

427

428

429

430

431

432

433

Considering that our measurement error ranges from 1 to $10 \%$, the mean errors obtained in the pixel to weight estimation are acceptable. For instance, on wheat bran our estimation error of $13 \%$ is only slightly higher than our measuring error (5-10\%). These errors are acceptable for our industrial context that requires absolute errors within $1 \%$. For our example recipe containing $3.75 \%$ of wheat bran this would correspond to a $0.48 \%$ absolute error (below the required error of $1 \%$ ). However, for recipes with more than $10 \%$ of wheat bran the error would be $1.3 \%$, slightly over $1 \%$. In such case we could still reduce the estimation error by averaging over different images, spreading out the ingredients or performing the study with a higher precision weight measuring device.

From literature, we could expect up to 30\% variation in flake thicknesses for oat (Rosentrater et al., 2018) and barley (Schwandt et al., 2017). Therefore, we could expect some weight variation between flakes even of the same size contributing to an increased weight estimation error. However, we have incurred in low errors of around $5 \%$ for both oat and barley flakes. Therefore, we hypothesize that by measuring several flakes simultaneously, we have also averaged out potential thickness variations between flakes.

\section{Band analysis with Genetic Algorithm and comparison to RGB}

The Genetic Algorithm is run together with our selected classification method (LDA+QDC). The results obtained are shown in Table 10, comparing the mean and minimum class accuracies per pixel for different band subsets.

Table 10: Band analysis and discrimination accuracy

\begin{tabular}{|l|r|l|c|c|}
\hline & \# bands & bands & Mean accuracy (\%) & Min accuracy (\%) \\
\hline All bands & 147 & $470-900 \mathrm{~nm}$ & 93.1 & 75.3 \\
\hline Best 15 & 15 & $470,492,500,518,553, \ldots 817 \mathrm{~nm}$ & 89.9 & 65.2 \\
\hline Best 5 & 5 & $500,567,624,773,884 \mathrm{~nm}$ & 88.1 & 54.1 \\
\hline Best 3 & 3 & $492,632,842 \mathrm{~nm}$ & 83.8 & 53.2 \\
\hline RGB & 3 & 3 broad bands & 76.9 & 35.9 \\
\hline
\end{tabular}

As we can see, using all 147 available bands provides the highest mean pixel accuracy for our test set $(93.1 \%)$ as well as the highest minimum pixel accuracy per class (75\% corresponding to millet). However, the number of bands can be reduced to, for instance, 15 bands without heavily compromising the mean accuracy ( 90\%). The selected bands show that the visible range is important, for instance to discriminate red millet from yellow millet. In the case of millet, the relevant pigments are carotenoids for yellow millet in the 450-480 nm range (Yano et al., 2017) and anthocyanins for red millet in the 450-600nm range (Kobayashi et al., 2006). Kong et al, (2013) have also shown that the selection of a reduced number of bands can still maintain high classification accuracy. In that study $100 \%$ accuracy was obtained for rice seed discrimination when all bands were used and around $90 \%$ accuracy when 12 selected bands were used. Boelt et al, (2018) and Carstensen et al, (2016) also achieved high discrimination accuracy above $90 \%$ with only 20 bands provided by their multiple LED system. Nevertheless, for some applications it may be preferable to have an initial higher number of bands available (such as 150 in our hyperspectral system) to maximize discrimination.

It is also interesting to notice that the best combination of 3 bands (492, 632 and $842 \mathrm{~nm})$ outperforms the use of RGB bands considerably, increasing both mean and minimum accuracies. Similar findings were obtained by (Vu et al., 2016) where rice seed variety discrimination is increased from $74 \%$ for RGB features to $84 \%$ with VNIR hyperspectral imaging.

The same classifier and spatial post-processing described for the hyperspectral image were applied to the RGB image. This results in high seed estimation error for some ingredients in Table 11: 1.72\% absolute error for millet, and $0.65 \%$ for sesame, corresponding to $2 \%$ real abundance. Therefore, for a sesame abundance of $4 \%$ this would result in $1.3 \%$ absolute error, above the maximum allowed. We can conclude that hyperspectral imaging clearly outperforms RGB making it a promising tool for accurate and fast multi-ingredient estimation. 
Table 11: Seed estimation accuracy for RGB broadband image

\begin{tabular}{|l|l|c|c|c|c|}
\hline Ingredient & $\begin{array}{l}\text { Percentage } \\
(\boldsymbol{\%})\end{array}$ & $\begin{array}{l}\text { Actual \# } \\
\text { seeds }\end{array}$ & $\begin{array}{l}\text { Estimated \# } \\
\text { seeds }\end{array}$ & $\begin{array}{l}\text { Relative } \\
\text { error (\%) }\end{array}$ & $\begin{array}{l}\text { Absolute } \\
\text { error (\%) }\end{array}$ \\
\hline Sunflower & 1.75 & 2 & 2 & 0.00 & 0.00 \\
\hline Linseed & 2.00 & 16 & 16 & 0.00 & 0.00 \\
\hline Corn & 1.75 & 5 & 4 & 20.00 & 0.35 \\
\hline Red millet & 2.00 & 14 & 14 & 0.00 & 0.00 \\
\hline Yellow millet & 2.00 & 22 & 4 & 81.81 & $\mathbf{1 . 6 3}$ \\
\hline Sesame & 2.00 & 37 & 25 & 32.43 & $\mathbf{0 . 6 4}$ \\
\hline Poppy seed & 1.75 & 113 & 103 & 8.84 & 0.15 \\
\hline
\end{tabular}

For non-seed ingredients such as wheat bran the classification accuracy with hyperspectral imaging is of $86 \%$ (see Table 5) and with RGB bands this decreases to 63\% accuracy. For this reason, we can also expect higher error in the weight estimation of non-seed ingredients since it is based on the pixel count.

\section{CONCLUSIONS}

Hyperspectral imaging in the VNIR range was shown to provide quantification of both seed and non-seed ingredients in multigrain wheat flour mixes in an automatic way and with higher accuracy than regular color imaging. Combination of spectral and spatial processing provides a total seed count with an absolute error below $1 \%$ for most ingredients. For non-seed ingredients (flakes and wheat) the estimation was done based on a total pixel count of the labelled pixels and resulted as well in the required accuracy in most cases.

This application requires relatively high spatial resolution to be able to correctly classify small ingredients, while still being able to cover a large enough area with a representative amount of different flour ingredients. For this reason, the new generation of VNIR hyperspectral cameras with high spectral and spatial resolution (up to 7 Mpixels) are considered suitable for this application. A comparison has been made with the discrimination accuracy of a hypothetical color camera with the same spatial resolution and measurement setup. Hyperspectral imaging, even with a reduced number of 15 bands, outperforms colour imaging by up to $15 \%$ in terms of the mean classification accuracy, which results in more accurate estimates for the ingredient fractions.

\section{ACKNOWLEDGMENTs}

This work was carried out in the context of the iFAST project with the support from Flanders' FOOD and the VLAIO (Agentschap Innoveren \& Ondernemen), research and innovation program under grant agreement No 140992.

\section{REFERENCES}

Amigo, J.M., Marti-Aluja, I. \& Gowen, A. (2013) Hyperspectral Imaging and Chemometrics: A Perfect Combination for the Analysis of Food Structure, Composition and Quality. Chemometrics in Food Chemistry Book, Elsevier, 2013.

Bakery products market- growth, trends and forecast (2019-2024) https://www.mordorintelligence.com/industryreports/bakery-products-market

Blanch, C., Saeys, W., \& Lambrechts, A. (2019). Hyperspectral Imaging for textile sorting in the VIS-NIR range. Journal of Spectral Imaging, 2019.

Boelt, B., Shrestha, S., Salimi, Z., Jorgensen, J.R., Nicolaisen, M \& Carstensen, J.M. (2018). Multispectral imaging - a new tool in seed quality assessment?. Seed Science Research, Vol 28, Special Issue 3 (Seeds as Systems).

Caporaso, N., Whitworth, Martin B., \& Fisk, Ian.D. (2018). Near-Infrared spectroscopy and hyperspectral imaging for nondestructive quality assessment of cereal grains. Applied Spectroscopy Reviews 2018, DOI: 10.1080/05704928.2018.1425214

Carstensen, J.M (2018). LED spectral imaging with food and agricultural applications. Proceedings of SPIE, the International Society for Optical Engineering, Vol. 10656.

Choudhary, R., Mahesh, S., Paliwal, J. \& Jayas, D.S. (2009). Identification of wheat classes using wavelet features from near infrared hyperspectral images of bulk samples. in Biosystems Engineering 102, www.elsevier.com/locate/issn/15375110

Feng, L., Zhu, S., Liu, F., He, Y., Bao, Y. \& Zhang, C. (2019). Hyperspectral imaging for seed quality and safety inspection: a review. Plant Methods (2019) 15:91

https://doi.org/10.1186/s13007-019-0476-y 
Fuller, D., Colledge, S., Murphy, C. \& Stevens, C.J. (2017). Sizing up cereal variation: patterns in grain evolution revealed in chronological and geographical comparison.

Ghuneim, A,

http://www.imageprocessingplace.com/downloads_V3/root_downloads/tutorials/contour_tracing_Abeer_George_Ghuneim/ connect.html

Giacco, R., et al, (2010). Effects of the regular consumption of wholemeal wheat foods on cardiovascular risk factors in healthy people. Nutr Metab Cardiovasc Dis 2010; 20:186-94.

Hai,V. et al, (2016). "Rice seed varietal purity inspection using hyperspectral imaging" Hyperspectral Imaging and Applications Conference, Coventry, United Kingdom.

Han, J., et al., (2012) “Data mining: concepts and techniques” https://doi.org/10.1016/C2009-0-61819-5

Hsu, C-W., et al. (2016) “A practical guide to support vector classification", available at https://www.csie.ntu.edu.tw/ cjlin/papers/guide/guide.pdf

Huang. H et al, (2014) "Recent Developments in Hyperspectral Imaging for Assessment of Food Quality and Safety", Journal of Sensors, April 2014.

Hunter Lab, CIE observer angle. https://support.hunterlab.com/hc/en-us/articles/203420099-CIE-Standard-Observers-andcalculation-of-CIE-X-Y-Z-color-values-AN-1002b

Kobayashi, T., Nagata, H., Goto, Y., Toyoda, H. \& Tallada, J. (2006) "Study on Anthocyanin Pigment Distribution Estimation for Fresh Fruits and Vegetables Using Hyperspectral Imaging. Part 2. Visualization of Anthocyanin Pigment Distribution of Strawberry (Fragaria x ananassa Duchesne)" in Shokubutsu Kankyo Kogaku, 2006.

Kong, W. et al, (2013). "Rice seed cultivar identification using near-infrared hyperspectral imaging and multivariate data analysis", in Sensors 2013, ISSN 1424-8220 www.mdpi.com/journal/sensors

Kurtulmus, F. et al, (2015) "Classification of pepper seeds using machine vision based on neural network" International Journal on Agriculture \& Biology Engineering, 2015.

Lorente, D. et al, (2011) "Recent Advances and Applications of Hyperspectral Imaging for Fruit and Vegetable Quality Assessment “, in Food and Bioprocess Technology · May 2011

Ma, F et al (2015) Discrimination of kernel quality characteristics for sunflower seeds based on multispectral imaging approach. Food Analytical Methods 8, 1629-1636.

Manley, M. et al, (2011), "Influence of grain topography on near infrared hyperspectral images", in Talanta Elsevier, 89223-230. www.elsevier.com/locate/talanta

Matlab, The Mathworks, Natick, 2015. https://mathworks.com

Mathison, G.W et al, (1997) "Effects of tempering and degree of processing of barley grain on the performance of bulls in the feedlot", Canadian journal of animal science, 1997.

Mishra,P ; et al, (2015) "Hyperspectral to multispectral imaging for detection of tree nuts and peanut traces in wheat flour", Journal of Spectral Imaging, June 2015.

Naes, T., et al, (2004). "A User-Friendly Guide to Multivariate Calibration and Classification.”

Patel, K.K. et al (2012), "Machine vision system: a tool for quality inspection of food and agricultural products", Journal of Food Science and Technology, 2012. https://www.ncbi.nlm.nih.gov/pmc/articles/PMC3550871/

Pearson, T.C, (2013) “Accurate seed sorting with machine vision”, https://www.vision-

systems.com/articles/2013/07/accurate-seed-sorting-with-machine-vision.html

\section{PerClass BV (2008-2019) http://perclass.com/perclass-toolbox/}

Pichette, J., et al, (2017) "Fast and compact internal scanning CMOS-based hyperspectral camera: the Snapscan", Proc. SPIE 10110, Photonic Instrumentation Engineering IV, (February 2017)

Rezaghli, F. et al, (2017) "Integration of fuzzy logic and computer vision in intelligent quality control of celiac-friendly products"

Rosentrater, K.A. et al, (2018) from Chapter 9: Breakfast Cereals in "Kent's technology of Cereals", 2018.) https://doi.org/10.1016/B978-0-08-100529-3.00009-8 
Sharkma, S. et al (2018), "Formulation of Functional Multigrain Bread and Evaluation of their Health Potential", International Journal of Current Microbiology and Applied Sciences ISSN: 2319-7706 Vol 7 Number 07 (2018)

Shelton, D. et al, (2004) "Wheat and Flour Testing Methods: A Guide to Understanding Wheat and Flour Quality". By the Wheat Marketing Center, Inc. Portland Oregon. ABD, 2004.

Schwandt, F. et al, (2017) "Flake density, roll diameter and flake moisture all influence starch availability of steam-flaked corn" in Kansas Agricultural Experiment Station Research Reports. 3. 10.4148/2378-5977.1356.

Smith, L. et al, (2002) “A tutorial on principal component analysis”, 2002.

www.cs.otago.ac.nz/cosc453/student.../principal_components.pdf

Sun, J. et al, (2016), "Classification of black beans using visible and near infrared hyperspectral imaging", in International Journal of Food Properties, 19:8, 1687-1695, DOI: 10.1080/10942912.2015.1055760

Szczypiński, P.M et al, (2015) Identifying barley varieties by computer vision. Computers and Electronics in Agriculture.

Talaei, M. et al (2013), "Healthy Bread Initiative: Methods, Findings, and Theories-Isfahan Healthy Heart Program", Journal of Health, Population and Nutrition, March 2013.

Wang, X. et al, (2018) "Near-infrared hyperspectral imaging for detection and quantification of azodicarbonamide in flour ", Journal of Science and Food Agriculture. 2018

Goodman, T.A (2012), Colour Design, Theories and Applications, International Standards for Colour, Pages 417-452

International Color Consortium 1999, sRGB Doc, IEC 61966-2-1:1999 http://www.color.org/chardata/rgb/srgb.xalter

Holland, J.H. (1992) Genetic Algorithms. Scientific American, 267. http://dx.doi.org/10.1038/scientificamerican0792-66

Vu, H., Tachtatzis, C., Murray, P., Harle, D., Dao, T.K., Atkinson, R., Le, T-L., Andonovic, A., \& Marshall, S. (2016) Spatial and spectral features utilization on a Hyperspectral imaging system for rice seed varietal purity inspection. 2016 IEEE RIVF International Conference on Computing \& Communication Technologies, Research, Innovation, and Vision for the Future.

Yano, A., Takakusagi, M., Oikawa, K., Nakajo, S. \& Sugawara, S. (2017) Xanthophyll levels in foxtail millet grains according to variety and harvesting time. Journal of Plant Production Science.

Yiu, T (2019). https://towardsdatascience.com/understanding-random-forest-58381e0602d2

Zhang, X et al, (2012). "Application of hyperspectral imaging and chemometric calibrations for variety discrimination of maize seeds" in Sensors, 2012, ISSN 1424-8220 www.mdpi.com/journal/sensors

Zhao, X et al, (2018) "Evaluation of Near-Infrared Hyperspectral Imaging for Detection of Peanut and Walnut Powders in Whole Wheat Flour", Journal of Applied Sciences, July 2018. 